\title{
Impedance Analysis Using High-resolution Impedance Manometry Facilitates Assessment of Pharyngeal Residue in Patients With Oropharyngeal Dysphagia
}

\author{
Tae Hee Lee, ${ }^{1}$ Joon Seong Lee, ${ }^{1 *}$ Su Jin Hong, ${ }^{2}$ Ji Sung Lee, ${ }^{3}$ Seong Ran Jeon, ${ }^{1}$ Wan Jung Kim, ${ }^{4}$ Hyun Gun Kim, ${ }^{1}$ \\ Joo Young Cho, ${ }^{1}$ Jin-Oh Kim, ${ }^{1}$ Jun-Hyung Cho, ${ }^{1}$ Mi-Young Kim ${ }^{1}$ and Soon Ha Kwon ${ }^{1}$ \\ ${ }^{1}$ Institute for Digestive Research, Digestive Disease Center Soonchunhyang University College of Medicine, Seoul, Korea; ${ }^{2}$ Department of \\ Internal Medicine, Soonchunhyang University College of Medicine, Bucheon, Gyeonggi-do, Korea; ${ }^{3}$ Biostatistical Consulting Unit, \\ Soonchunhyang University College of Medicine, Seoul, Korea; and ${ }^{4}$ Department of Internal Medicine, Soonchunhyang University Hospital, \\ Gumi, Gyeongsangbuk-do, Korea
}

\section{Background/Aims}

Impedance analysis using high-resolution impedance manometry (HRIM) enables the recognition of pharyngeal residue in patients with oropharyngeal dysphagia. The aims of this study were to evaluate appropriate criteria for impedance analysis in a large patient cohort, as well as the diagnostic accuracy and agreement of analysis performed by HRIM trainees.

\section{Methods}

We reviewed 33 controls (13 males; median age, 61.2 years) and 104 oropharyngeal dysphagia patients (61 males; median age, 70.4 years) who underwent a flexible endoscopic evaluation of swallowing study (FEES) and HRIM. Two experts compared the pharyngeal residue on FEES and impedance color pattern at 1,000, 1,500 and 2,000 $\Omega$ of the impedance bar. Three trainees were given a 60 minutes tutorial to determine the diagnostic accuracy and agreement of this analysis.

\section{Results}

The diagnostic sensitivity of experts for predicting liquid residue was $73.1 \%$ for $1,000 \Omega, 96.2 \%$ for $1,500 \Omega$ and $100 \%$ for $2,000 \Omega$. Significantly higher sensitivity was observed at 1,500 $\Omega$ compared to $1,000 \Omega(P<0.001)$. The diagnostic specificity of experts for liquid residue was $98.3 \%$ for $1,000 \Omega, 96.6 \%$ for $1,500 \Omega$ and $83.1 \%$ for $2,000 \Omega$. There was a higher specificity at $1,500 \Omega$ compared to $2,000 \Omega(P=0.008)$. The $\kappa$ value among the 3 trainees was 0.89 and the diagnostic accuracy of the trainees for liquid residue was comparable to that of the experts.

Received: February 1, 2014 Revised: March 17, 2014 Accepted: April 8, 2014

(c) This is an Open Access article distributed under the terms of the Creative Commons Attribution Non-Commercial License (http://creativecommons. org/licenses/by-nc/3.0) which permits unrestricted non-commercial use, distribution, and reproduction in any medium, provided the original work is properly cited.

*Correspondence: Joon Seong Lee, MD, PhD

Institute for Digestive Research, Digestive Disease Center, Soonchunhyang University Hospital, Deasagwan-gil 22, Yongsan-gu, Seoul, Korea

Tel: +82-2-709-9863, Fax: +82-2-795-3687, E-mail: joonlee@schmc.ac.kr

Financial support: This work was supported in part by the Soonchunhyang University Research Fund.

Conflicts of interest: None.

Author contributions: Tae Hee Lee and Joon Seong Lee designed the study and wrote the manuscript; Su Jin Hong, Seong Ran Jeon, Wan Jung Kim, Hyun Gun Kim, Joo Young Cho, Jin-Oh Kim, Jun-Hyung Cho1, Mi-Young Kim and Soon Ha Kwon reviewed the manuscript; Ji Sung Lee performed stastistical analysis.

ORCID: Tae Hee Lee, http://orcid.org/0000-0003-3049-8252. 


\section{Conclusions}

The impedance analysis at 1,500 $\Omega$ provides more accurate information for the detection of liquid residue, irrespective of the level of expertise.

(J Neurogastroenterol Motil 2014;20:362-370)

\section{Key Words}

Deglutition disorders; Diagnosis; Pharynx

\section{Introduction}

Oropharyngeal dysphagia (OPD) is significantly associated with nutritional deficiency and aspiration pneumonia, which can lead to fatal results. ${ }^{1,2}$ Laboratory evidence of OPD can assist prevention of serious consequences in OPD patients. Several studies reported the utility of high-resolution impedance manometry (HRIM) for evaluation of oropharyngeal dysphagia. ${ }^{3-11}$ The risk of post-deglutitive aspiration was negligible for patients without pharyngeal retention and very high for patients with moderate to severe pharyngeal residue, ${ }^{12}$ which suggested that post-swallow residue was an indicator of interest when evaluating patients with OPD.

Omari et al ${ }^{13,14}$ reported that the swallow risk index (SRI) or the integral of ratio of nadir impedance to post-swallow impedance ( $\mathrm{i} \mathrm{Zn} / \mathrm{Z}$ ) was useful in predicting ineffective pharyngeal swallowing relevant to post-swallow pharyngeal residue. However, these new quantitative approaches have not been incorporated into current HRIM analysis software packages and thus, are not available to clinicians. Therefore, a simpler measurement method is needed for prediction of pharyngeal residue.

We have previously evaluated the utility of HRIM-based qualitative impedance for detection of pharyngeal residue in small study of 20 healthy volunteers and 10 OPD patients that used a pre-set cut-off of $1,000 \Omega,{ }^{15}$ which was arbitrarily selected for color impedance analysis. We have observed a linear pattern (vertical line) under a value of $1,000 \Omega$ in the impedance color bar of the subjects without pharyngeal residue in the videofluoroscopic swallowing study (VFSS), while a stasis pattern (a horizontal square) was seen in the subjects with pharyngeal residue. The percentage agreement and $\kappa$ value for detecting pharyngeal residue between the VFSS and impedance color pattern were 100\% and 1.0, respectively. Therefore, we reported that HRIM- based visual pattern analysis of the impedance color enabled accurate recognition of pharyngeal residue.
However, larger studies using these approaches are needed to assess their reproducibility and validate the method in OPD patients. Therefore, the aim of this study was to evaluate appropriate criteria for impedance analysis in a larger patient cohort. In addition, we investigated whether this methodology allows acceptable diagnostic accuracy and agreement among non-experts.

\section{Materials and Methods}

\section{Subjects}

This study included 104 OPD patients and 33 asymptomatic subjects. To select the OPD patient group, a cohort of 150 consecutive stroke patients undergoing HRIM from January 2012 to December 2012 was screened as study subjects.

Patients were selected for inclusion after excluding individuals with unavailable information regarding OPD severity; various artifacts of impedance color; $>2$ days from HRIM to a flexible endoscopic evaluation of swallowing study (FEES); and significant distal esophageal motility disorders (e.g., achalasia and diffuse esophageal spasm). After applying these criteria, 104 OPD patients with stroke (61 males; mean age, 70.4 years; age range, 26-88 years) were included in this study. The American Speech-Language-Hearing Association (ASHA) National Outcome Measurement System (NOMS) swallowing scale was used to assess the severity of OPD. ${ }^{16}$ The ASHA NOMS swallowing level is a measurement of dysphagia with a severity from 7 to 1 which takes into account the supervision required and diet level that intuitively reflects patient's functional status.

For comparison, we reviewed another database of 40 asymptomatic subjects to assess normal parameters of HRIM for upper esophageal sphincter relaxation. These subjects included 33 controls (13 males; mean age, 61.2 years; age range, 50-86 years) that underwent both HRIM and a FEES on the same day. The study protocol was approved by the Institutional Review Board of Soonchunhyang University Seoul Hospital, College of Medi- 
cine, Seoul, Korea.

\section{High-resolution Impedance Manometry Study Protocol}

All subjects underwent an HRIM study by 2 independent examiners (JSL and THL) after at least a 6-hour fast. The HRIM catheter was $4.2 \mathrm{~mm}$ in diameter, with 36 circumferential pressure sensors at $1-\mathrm{cm}$ intervals and 18 impedance channels at 2-cm intervals (Given Imaging, Los Angeles, CA). The HRIM study was performed using both a standard and a modified protocol. In the standard protocol, the catheter was passed transnasally into the esophagus to a depth of $60 \mathrm{~cm}$. The tip was in the cardia of the stomach, and the zero mark on the probe was located at the channel used for LES analysis. After 5 minutes of adaptation in the sitting position, subjects were asked to perform 10 saline swallows ( $5 \mathrm{~mL}$ each) spaced at 20 -second intervals. Bolus transits of the pharyngeoesophageal (PE) segment and manometric patterns of the entire pharynx were not able to be assessed using the HRIM standard protocol. Therefore, the HRIM catheter was pulled $10 \mathrm{~cm}$ back and 5 saline swallows ( $5 \mathrm{~mL}$ each) spaced at 20-second intervals were repeated in a modified protocol.

Our assessment of bolus transits of the PE segment was similarly performed as described previously. ${ }^{15}$ Impedance topography using the modified protocol defined a region of interest (ROI) as the horizontal portion between the 2 portions, corresponding to the beginning point of the pre-upper esophageal sphincter (UES) swallow peak and the ending point of the post-UES peak in the impedance contour view, respectively. The
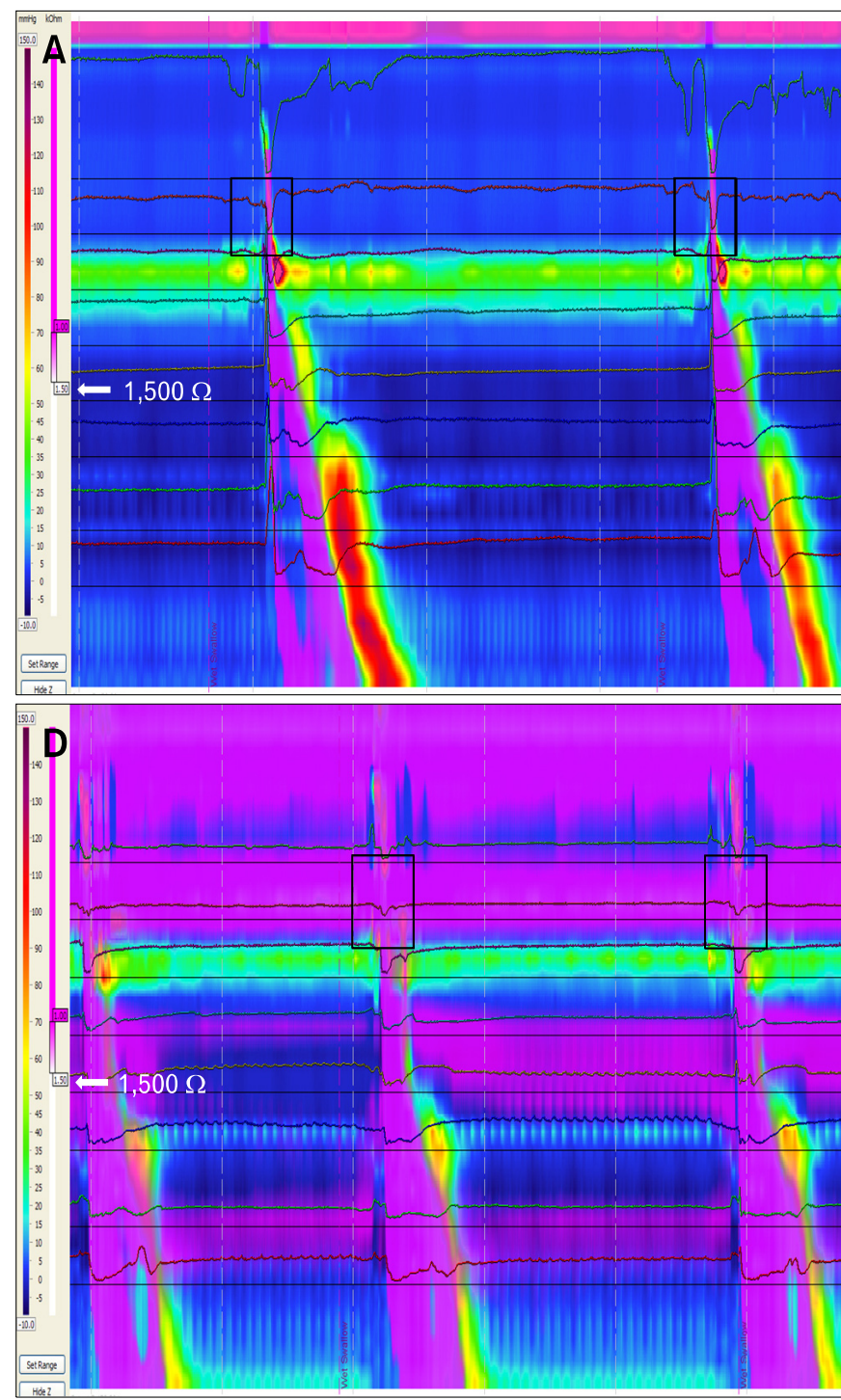
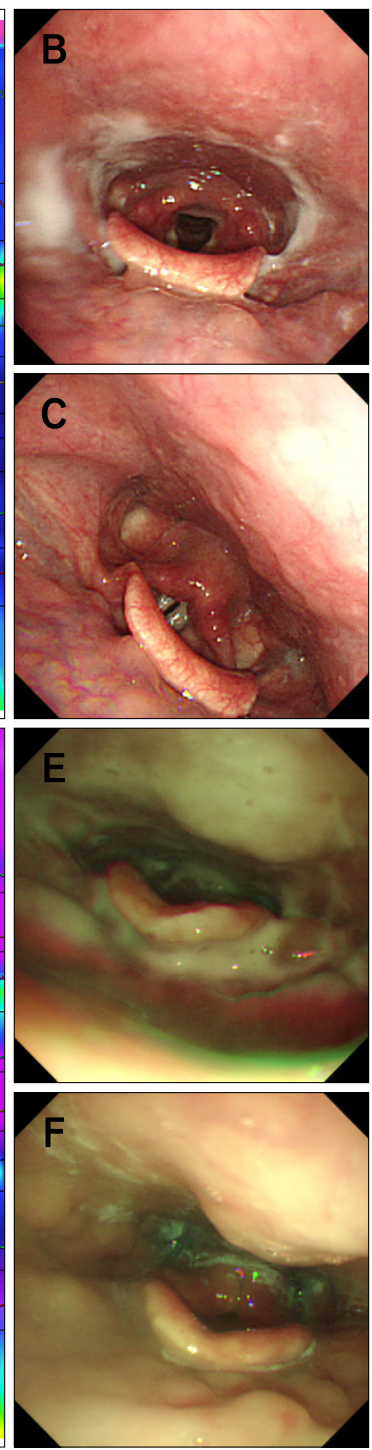

Figure 1. Vertical linear vs. horizontal square patterns during impedance contour analysis of the pharyngoesophageal segment. (A) A vertical linear pattern of bolus transit in the region of interest (black-lined box) was observed in a 51-year-old female with oropharyngeal dysphagia. (B) A viscous material partially coated the pharyngeal wall but retention was not visible at the valleculae and pyriform sinuses after a $5 \mathrm{~mL}$ yogurt swallow during flexible endoscopic evaluation of swallowing (FEES). (C) Pharyngeal residue was not noted after a 5 $\mathrm{mL}$ saline swallow during FEES. (D) A horizontal square pattern of bolus transit in the region of interest (black-lined box) was seen in a 68-year-old female with oropharyngeal dysphagia. (E) A large amount of pharyngeal residue was visible after a $5 \mathrm{~mL}$ yogurt swallow during FEES. (F) A medium amount of residue was observed at the pyriform sinuses after a $5 \mathrm{~mL}$ saline swallow during FEES. 
vertical portion of the ROI was the region between the 2 portions, corresponding to the point of mesohypopharyngeal contraction and the point of UES in the impedance contour view. Impedance tracing was obtained by marking retrograde drops in impedance propagating 2 impedance segments across the UES, and bolus transits of the PE segment were assessed by interpreting the impedance purple color contours superimposed over the tracings within the ROI. The superimposed impedance color contour patterns, shown in purple, were classified as the vertical linear and horizontal square impedance patterns at $1,500 \Omega$ (Fig. 1). A vertical linear pattern was defined when the downward straight shaped purple color appeared and then disappeared in the ROI. In contrast, the horizontal square pattern was defined when the impedance color contour pattern within the ROI did not meet the criteria of the vertical linear pattern; for example, the purple color was spread over the ROI or did not appear in an on-again, off-again pattern. These analyses were also performed at $1,000 \Omega$ and 2,000 $\Omega$ (Fig. 2).
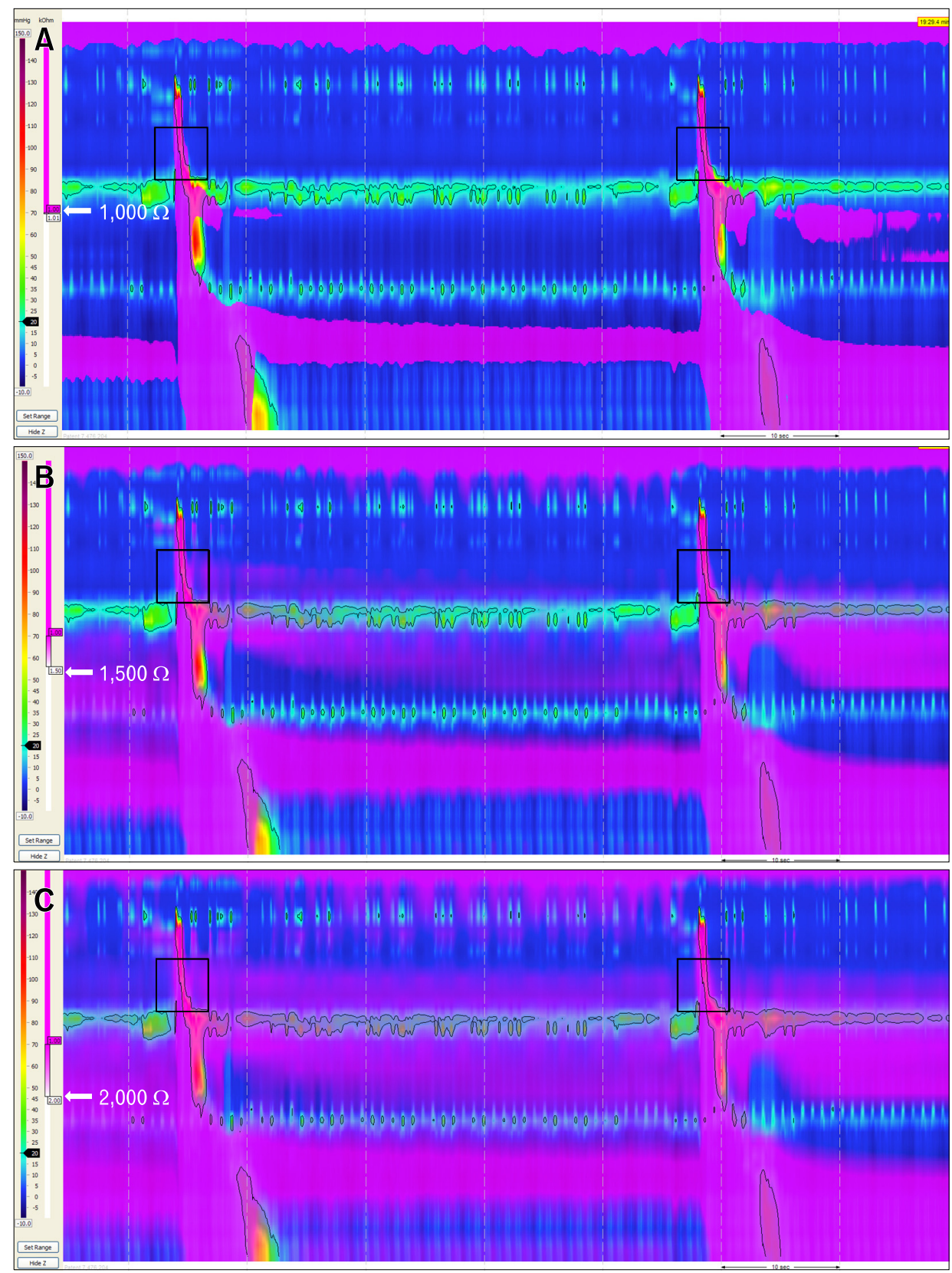

Figure 2. Different impedance color patterns based on three impedance bars. Bolus transit of pharyngeoesophageal segment was seen as a vertical linear pattern at $1,000 \Omega$ (A) but as a horizontal square pattern at $1,500 \Omega(\mathrm{B})$ and 2,000 $\Omega(\mathrm{C})$. 
Two experts (JSL and THL) performed these analyses for 137 HRIM patients (including 104 OPD and 33 controls) in a consensus reading. The expert physicians were blinded to the subjects' symptoms and FEES findings.

Three fellows (EJP, JNK and MJK) with various levels of HRIM knowledge were trained at the Institute for Digestive Research, Soonchunhyang University, College of Medicine in Seoul, Republic of Korea. Only one of these trainees had an advanced level of HRIM knowledge, which consisted of participation in a study involving interpretation of distal esophageal motility. All of 3 trainees were given a 60-minute tutorial by one (THL) of the study investigators with experience in this impedance color analysis. One day following the tutorial, the diagnostic accuracy and agreements for this analysis among the 3 trainees, who were blinded to the subjects' symptoms and FEES findings, were evaluated from the data of 137 HRIM patients (104 OPD and 33 controls).

\section{Flexible Endoscopic Evaluation of Swallowing Study Protocol}

FEES was performed by an experienced endoscopist (THL). A thin video gastroscope (Olympus GIF-XP 260, Olympus, Tokyo, Japan), with a $6.5 \mathrm{~mm}$ insertion tube, $2.0 \mathrm{~mm}$ channel and a working length of $1,030 \mathrm{~mm}$ was used during FEES. The endoscope was inserted through the nostril and placed between the end of the soft palate and the epiglottis. The patient was then allowed a 1-minute rest to adapt to the presence of the laryngoscope and prepare for the testing. The examination consisted of anatomic and physiologic assessment (including velar and laryngopharyngeal anatomy, movement and sensation) and the direct examination of swallowing test diets. When clinically indicated, an entire anatomic assessment of the esophagus and stomach was performed. For the FEES test diets, we first used 5 $\mathrm{mL}$ yogurt as a viscous food, followed by $5 \mathrm{~mL}$ indigo carmine dye mixed with water as liquid food, which enabled visualization as part of the FEES protocol adopted in the hospital. The entire clinical procedure was recorded on video for analysis of the procedure by the endoscopist (THL).

Pharyngeal residue was usually defined as retention of the entire given materials in the valleculae or pyriform sinuses after the swallow. However, double swallows are common in normal subjects, as well as in OPD patients. A previous study suggested that a small amount of residue, estimated at no more than $10-15 \%$ of the entire bolus, after the first swallow should be considered as a normal finding. ${ }^{16}$ Therefore, we defined pharyngeal residue in valleculae or pyriform sinuses as follows: (1) medium to large amount of residue after the first swallow and (2) small amount of residue even after a double swallow. The pharyngeal residue seen during either liquid or viscous swallowing on the FEES was considered the gold standard when we assessed the diagnostic accuracy of impedance color analysis for liquid and viscous residue.

\section{Statistical Methods}

Differences in impedance patterns between controls and OPD patients were assessed with the $\chi^{2}$ test. The ASHA NOMS swallowing level scale was compared between vertical linear and horizontal square impedance patterns using the Wilcoxon rank-sum test. Diagnostic accuracies of impedance analysis were compared among the three impedance bars using the exact McNemar test. The $\mathrm{K}$ value for classifying the impedance color pattern among the trainees was assessed using the SPSS ver. 12.0 software (SPSS, Chicago, IL, USA). Differences in ASHA NOMS scales between subjects with vertical linear and horizontal square impedance patterns were assessed with the Mann-Whitney U-test. A $P$-value of $<0.05$ indicated significance.

\section{Results}

\section{Pharyngeal Residue on Flexible Endoscopic Evaluation of Swallowing Study}

The FEES revealed liquid residue in six (18.2\%) controls, but no viscous residue was seen in any control. Liquid and viscous residue were observed in $72(69.2 \%)$ and 75 (72.1\%) of the OPD patients, respectively.

\section{Impedance Patterns Between Control and Oropharyngeal Dysphagia Patients}

A significant horizontal square pattern was observed in OPD patients, irrespective of the impedance bar, compared to controls (Table 1). In controls, the vertical linear pattern decreased from 1,000 (93.9\%) to 1,500 (84.9\%) to $2,000 \Omega$ (66.7\%), while the horizontal square pattern of OPD patients increased from 1,000 (53.9\%) to 1,500 (69.2\%) to $2,000 \Omega(74 \%)$.

\section{American Speech-Language-Hearing Association National Outcome Measurement System Swallowing Level Scale According to Impedance Pattern}

The ASHA NOMS swallowing level scale was significantly 
lower in the horizontal square pattern, irrespective of impedance bar, compared to the vertical linear pattern (Table 2). At 2,000 $\Omega$, subjects with a vertical linear pattern had a significantly higher $(P$ $<0.001)$ median ASHA NOMS swallowing level (7, range 5-7), compared to the horizontal square pattern (5, range 3-6). At $1,500 \Omega$, subjects with a vertical linear pattern had a significantly higher $(P<0.001)$ median ASHA-NOMS swallowing level (7, range $5-7)$, compared to the horizontal square pattern (4, range $3-5)$. At $1,000 \Omega$, subjects with a vertical linear pattern had a significantly higher $(P<0.001)$ median ASHA-NOMS swallowing level (6, range 5-7), compared to the horizontal square pattern (4, range 2-5).

\section{Diagnostic Accuracy of Liquid Residue}

Table 3 shows the diagnostic accuracy of impedance pattern analysis based on the three impedance bars. At 1,000 $\Omega$, diagnostic sensitivity of $73.1 \%$, specificity of $98.3 \%$, positive likelihood ratio of 43.1 , negative likelihood ratio of 0.27 , positive predictive value of $98.3 \%$ and negative predictive value of $73.4 \%$

Table 1. Impedance Color Patterns in the Control and Oropharyngeal Dysphagia Groups

\begin{tabular}{lccc}
\hline & Control $(\mathrm{n}=33)$ & OPD $(\mathrm{n}=104)$ & $P$-value \\
\hline $2,000 \Omega$ & & & $<0.001$ \\
Vertical linear & $22(66.7 \%)$ & $27(26.0 \%)$ & \\
Horizontal square & $11(33.3 \%)$ & $77(74.0 \%)$ & \\
$1,500 \Omega$ & & & $<0.001$ \\
Vertical linear & $28(84.9 \%)$ & $32(30.8 \%)$ & \\
Horizontal square & $5(15.2 \%)$ & $72(69.2 \%)$ & \\
$1,000 \Omega$ & & & $<0.001$ \\
Vertical linear & $31(93.9 \%)$ & $48(46.2 \%)$ & \\
Horizontal square & $2(6.1 \%)$ & $56(53.9 \%)$ & \\
\hline
\end{tabular}

$P$-value by $\chi^{2}$ test. were noted. At $1,500 \Omega$, diagnostic sensitivity of $96.2 \%$, specificity of $96.6 \%$, positive likelihood ratio of 28.4 , negative likelihood ratio of 0.04 , positive predictive value of $97.4 \%$, and negative predictive value of $95 \%$ were noted. At $2,000 \Omega$, diagnostic sensitivity of $100 \%$, specificity of $83.1 \%$, positive likelihood ratio of 5.9 , negative likelihood ratio of zero, positive predictive value of $88.6 \%$ and negative predictive value of $100 \%$ were noted.

The sensitivity was significantly higher, at $1,500 \Omega$, compared to $1,000 \Omega(P<0.001)$. However, there was no significant difference in sensitivity between $1,500 \Omega$ and 2,000 $\Omega$. A significantly higher specificity was observed at $1,500 \Omega$, compared to $2,000 \Omega(P=0.008)$, while the specificities at $1,500 \Omega$ and $1,000 \Omega$ were comparable.

Table 2. American Speech-Language-Hearing Association National Outcome Measurement System Swallowing Level Scale According to Impedance Pattern

\begin{tabular}{lcc}
\hline & $\begin{array}{c}\text { ASHA NOMS } \\
\text { swallowing level scale }\end{array}$ & $P$-value \\
\hline $2,000 \Omega$ & $7(5-7)$ & $<0.001$ \\
Vertical linear & $5(3-6)$ & \\
Horizontal square & & $<0.001$ \\
$1,500 \Omega$ & $7(5-7)$ & \\
Vertical linear & $4(3-5)$ & $<0.001$ \\
Horizontal square & & \\
$1,000 \Omega$ & $6(5-7)$ & \\
Vertical linear & $4(2-5)$ & \\
Horizontal square & & \\
\hline
\end{tabular}

ASHA NOMS, American Speech-Language-Hearing Association National Outcome Measurement System.

Data are presented as median (interquartile range). $P$-value by Wilcoxon rank-sum test.

Table 3. Diagnostic Accuracies for Liquid Residue According to the 3 Impedance Bars

\begin{tabular}{lccc}
\hline & $1,000 \Omega$ & $1,500 \Omega$ & $2,000 \Omega$ \\
\hline Sensitivity $(95 \% \mathrm{CI})$ & $73.1 \%(61.8-82.5 \%)$ & $96.2 \%^{\mathrm{a}}(89.2-99.2 \%)$ & $100.0 \%^{\mathrm{b}}(95.4-100.0 \%)$ \\
Specificity (95\% CI) & $98.3 \%(90.9-100.0 \%)$ & $96.6 \%^{\mathrm{c}}(88.3-99.6 \%)$ & $83.1 \%^{\mathrm{d}}(71.0-91.6 \%)$ \\
Positive LR (95\% CI) & $43.10(6.20-302.40)$ & $28.40(7.30-110.80)$ & $5.90(3.40-10.40)$ \\
Negative LR (95\% CI) & $0.27(0.19-0.40)$ & $0.04(0.01-0.12)$ & $0.00(0.00-0.00)$ \\
Positive PV (95\% CI) & $98.3 \%(90.8-100.0 \%)$ & $97.4 \%(90.9-99.7 \%)$ & $88.6 \%(80.1-94.4 \%)$ \\
Negative PV (95\% CI) & $73.4 \%(62.3-82.7 \%)$ & $95.0 \%(86.1-99.0 \%)$ & $100.0 \%(92.8-100.0 \%)$ \\
\hline
\end{tabular}

LR, likelihood ratio; $\mathrm{PV}$, predictive value.

Significant differences assessed using the exact McNemar test.

${ }^{\mathrm{a}}$ Significantly different from $1,000 \Omega(P<0.001)$ but not different from $2,000 \Omega(P=0.250),{ }^{\mathrm{b}}$ Significantly different from $1,000 \Omega(P<0.001),{ }^{\mathrm{c}}$ Significantly different from $2,000 \Omega(P=0.008)$ but not different from $1,000 \Omega(P>0.999)$, ${ }^{\mathrm{d}}$ Significantly different from $1,000 \Omega(P=0.004)$. 
Table 4. Diagnostic Accuracy for Viscous Residue Detection Based on 3 Impedance Bars

\begin{tabular}{lccc}
\hline & $1,000 \Omega$ & $1,500 \Omega$ & $2,000 \Omega$ \\
\hline Sensitivity (95\% CI) & $68.0 \%(56.2-78.3 \%)$ & $85.3 \%^{\mathrm{a}}(75.3-92.4 \%)$ & $88.0 \%^{\mathrm{b}}(78.4-94.4 \%)$ \\
Specificity (95\% CI) & $88.7 \%(78.1-95.3 \%)$ & $79.0 \%^{\mathrm{c}}(66.8-88.3 \%)$ & $64.5 \%^{\mathrm{d}}(51.3-76.3 \%)$ \\
Positive LR (95\% CI) & $6.00(3.00-12.30)$ & $4.10(2.50-6.70)$ & $2.50(1.80-3.50)$ \\
Negative LR (95\% CI) & $0.36(0.26-0.51)$ & $0.19(0.11-0.33)$ & $0.19(0.10-0.35)$ \\
Positive PV (95\% CI) & $87.9 \%(76.7-95.0 \%)$ & $83.1 \%(72.9-90.7 \%)$ & $75.0 \%(64.6-83.6 \%)$ \\
Negative PV (95\% CI) & $69.6 \%(58.3-79.5 \%)$ & $81.7 \%(69.6-90.5 \%)$ & $81.6 \%(68.0-91.2 \%)$ \\
\hline
\end{tabular}

LR, likelihood ratio; PV, predictive value.

Significant differences assessed using the exact McNemar test.

${ }^{\mathrm{a}}$ Significantly different from $1,000 \Omega(P<0.001)$ but not different from $2,000 \Omega(P=0.500)$, ${ }^{\mathrm{b}}$ Significantly different from $1,000 \Omega(P<0.001)$, ${ }^{\mathrm{c}}$ Significantly different from $1,000 \Omega(P=0.031)$ and $2,000 \Omega(P=0.004),{ }^{\text {d }}$ Significantly different from $1,000 \Omega(P<0.001)$.

Table 5. Diagnostic Accuracy of Liquid Residue Detection Among 3 Trainees

\begin{tabular}{lccc}
\hline & Trainee A & Trainee B & Trainee C \\
\hline Sensitivity (95\% CI) & $93.5 \%(85.5-97.9 \%)$ & $97.4 \%(90.9-99.7 \%)$ & $92.2 \%(83.8-97.1 \%)$ \\
Specificity (95\% CI) & $98.3 \%(91.1-100.0 \%)$ & $98.3 \%(91.1-100.0 \%)$ & $98.3 \%(91.1-100.0 \%)$ \\
Positive LR (95\% CI) & $56.10(8.00-392.20)$ & $58.40(8.40-408.30)$ & $55.30(7.90-386.80)$ \\
Negative LR (95\% CI) & $0.07(0.03-0.15)$ & $0.03(0.01-0.10)$ & $0.08(0.04-0.17)$ \\
Positive PV (95\% CI) & $98.6 \%(92.6-100.0 \%)$ & $98.7 \%(92.9-100.0 \%)$ & $98.6 \%(92.5-100.0 \%)$ \\
Negative PV (95\% CI) & $92.2 \%(82.7-97.4 \%)$ & $96.7 \%(88.7-99.6 \%)$ & $90.8 \%(81.0-96.5 \%)$ \\
\hline
\end{tabular}

$\mathrm{LR}$, likelihood ratio; $\mathrm{PV}$, predictive value.

\section{Diagnostic Accuracy of Viscous Residue}

Comparative results among the three impedance bars are shown in Table 4 . At $<1,000 \Omega$, diagnostic sensitivity of $68 \%$, specificity of $88.7 \%$, positive likelihood ratio of 6 , negative likelihood ratio of 0.36 , positive predictive value of $87.9 \%$ and negative predictive value of $69.6 \%$ were reported. At $1,500 \Omega$, diagnostic sensitivity of $85.3 \%$, specificity of $79 \%$, positive likelihood ratio of 4.1 , negative likelihood ratio of 0.19 , positive predictive value of $83.1 \%$ and negative predictive value of $81.7 \%$ were noted. At $2,000 \Omega$, diagnostic sensitivity of $88 \%$, specificity of $64.5 \%$, positive likelihood ratio of 2.5 , negative likelihood ratio of 0.19 , positive predictive value of $75 \%$ and negative predictive value of $81.6 \%$ were noted.

Significantly higher sensitivity $(P<0.001)$ was observed at $1,500 \Omega$ compared to $1,000 \Omega$. However, there was no significant difference in sensitivity between 1,500 and 2,000 $\Omega$. Specificity was higher at $1,500 \Omega$ compared to $2,000 \Omega(P=0.004)$, and at $1,000 \Omega$ compared to $1,500 \Omega(P=0.031)$.

\section{Diagnostic Agreement and Accuracy Among 3 Learners}

The $\kappa$ value for inter-observer agreement among the 3 trainees was 0.89 . The diagnostic accuracy of the three learners is compared in Table 5. In one trainee (JNK), impedance analysis at $1,500 \Omega$ showed diagnostic sensitivity of $93.5 \%$, specificity of $98.3 \%$, positive likelihood ratio of 56.1 , negative likelihood ratio of 0.07 , positive predictive value of $98.6 \%$, and negative predictive value of $92.2 \%$. In another trainee (EJP), impedance analysis at $1,500 \Omega$ showed diagnostic sensitivity of $97.4 \%$, specificity of $98.3 \%$, positive likelihood ratio of 58.4 , negative likelihood ratio of 0.03 , positive predictive value of $98.7 \%$ and negative predictive value of $96.7 \%$. In the last trainee (MJK), impedance analysis at $1,500 \Omega$ showed diagnostic sensitivity of $92.2 \%$, specificity of $98.3 \%$, positive likelihood ratio of 55.3 , negative likelihood ratio of 0.08 , positive predictive value of $98.6 \%$ and negative predictive value of $90.8 \%$.

\section{Discussion}

Analysis that uses impedance tracing of the PE segment presents an overwhelming challenge. The impedance criteria for 
detecting failure of bolus clearance in the pharynx remains to be defined. Standard impedance analysis defines the failure of bolus clearance as $50 \%$ from baseline. This approach has been accepted for assessing esophageal bolus transit but is problematic in the pharynx. Szczesniak et al, ${ }^{17}$ reported that the optimal impedance criteria defining bolus presence across the PE segment differ from those adopted for the esophagus. However, the robustness of this approach needs to be assessed, particularly in clinical situations.

We hypothesized that an analysis of the visual pattern of the impedance might lead to a better understanding of incomplete pharyngeal swallowing. A purple shaded area of impedance, which indicates bolus presence, detects the conductivity of the bolus swallows while pharyngeal residue is representative of incomplete pharyngeal swallowing. In this way, complete pharyngeal bolus transit may be observed with the onset of contraction at each pharyngeal level corresponding to the clearance of purple colorization and upward inflection in the impedance tracings. In a pilot study of a small number of patients, we found that impedance color analysis (vertical linear vs. horizontal square) helped to identify the presence of pharyngeal residue. ${ }^{15}$

The results of this larger study demonstrated that impedance color analysis (vertical linear vs. horizontal square) at 1,500 $\Omega$ provides information on liquid and viscous residue. During liquid swallow, analysis at 1,500 $\Omega$ showed high sensitivity of $96.2 \%$ and specificity of $96.6 \%$. Our subjective analysis appears to be a better assessment of pharyngeal residue compared to objective analysis (e.g., SRI). Omari et al, ${ }^{13}$ reported that an average SRI of 9 predicted residue with $75 \%$ sensitivity and $80 \%$ specificity using various volumes of semi-solid residue containing $1 \% \mathrm{NaCl}$ to enhance conductivity during HRIM examination. In contrast, $5 \mathrm{~mL}$ of $0.9 \% \mathrm{NaCl}$ was administered during our HRIM examination. Thus, differences in the diagnostic accuracies between these studies may have resulted from the use of different swallowed materials.

Diagnostic sensitivity and specificity for predicting viscous residue were lower (85.3\% and 79\%, respectively), although acceptably accurate in our study. Impedance is inversely related to electrical conductivity, thereby intraesophageal contents with high ionic concentrations have a low impedance, as opposed to the esophageal mucosa or air. ${ }^{18}$ Swallowing a viscous material might leave a coating on the pharyngeal mucosa, thereby influencing the assessment of bolus movement in subjects without ineffective swallowing. That is why the diagnostic accuracy is lower with a viscous residue, compared to a liquid residue. Further- more, our subjects were not asked to swallow viscous material during the HRIM examination, but to swallow saline. When we evaluated the diagnostic accuracy of viscous residue using impedance color analysis, the gold standard was pharyngeal residue on FEES during the $5 \mathrm{~mL}$ yogurt (viscous) swallow. Therefore, the lower diagnostic accuracy of a viscous residue was presumably due to the difference in the material swallowed in the FEES and HRIM examinations.

However, it should be noted that the diagnostic sensitivity and specificity of our study for predicting viscous residue were comparable to those of Omari et al, ${ }^{13}$ for predicting a semi-solid bolus. This difference might be due in part to the use of different standard methods between the 2 studies since we used FEES while Omari et al ${ }^{13}$ used a VFSS. FEES was reported to be more sensitive than VFSS in the assessment of penetration, aspiration and pharyngeal residue. ${ }^{19,20}$

Our data suggest that horizontal square pattern on impedance analysis is reflective of higher OPD severity, compared to a vertical linear pattern. At 1,500 $\Omega$, subjects with a vertical linear pattern had a significantly higher median ASHA NOMS swallowing level (7) compared to a horizontal square pattern (4). HRIM enables visualization of the mechanical dynamics by interpreting the interactions of pressure gradients. To our knowledge, HRIM helps clarify the pathogenesis of OPD. ${ }^{3,415}$ Given together, additional performance of color impedance analysis might improve the comprehensive assessment of HRIM for OPD patients.

This approach provided a reproducible and accurate avenue for detection of pharyngeal residue among trainees. The strength of the inter-observer agreement was almost perfect, according to the Landis and Koch criteria. ${ }^{21}$ Diagnostic accuracies of the three trainees were comparable to that of the two experts. This implies that this new approach might hold great appeal for clinicians because it is easy to practice.

The strength of our study is that qualitative analysis of impedance pattern was performed in a large cohort of OPD patients with strokes compared to controls, while previous studies had used a small sample size. However, there were several limitations to the present study. First, the FEES and HRIM determinations were not performed simultaneously, which might have biased the retrospective analysis. Second, the assessment of diagnostic accuracy for viscous residue was performed by evaluation of the HRIM impedance pattern during liquid swallowing. Third, the effect of different bolus volumes on impedance pattern was not evaluated. Differences in pharyngeal pressures and the timing of 
key pressure events have been detected across varying bolus volumes. ${ }^{22}$ At larger volumes, the pattern of greater velopharyngeal pressure and increased duration of UES opening is characteristic of typical swallowing in normal subjects. Therefore, we should assess whether a larger volume might affect the horizontal square impedance pattern in OPD patients compared to the vertical linear pattern at smaller volume. Finally, qualitative pattern analysis of PE segment impedance was subjective and may thus be associated with significant intra- and inter-observer variability, although the $\kappa$ values for inter-observer agreement were near perfect in our study.

In conclusion, our results add to the growing body of evidence demonstrating the value of HRIM in the evaluation of OPD. Impedance analysis at $1,500 \Omega$ provided reliable information for the detection of liquid and viscous, irrespective of the level of expertise. This new approach could contribute to the widespread understanding of OPD among gastroenterologists.

\section{References}

1. Guyomard V, Fulcher RA, Redmayne O, Metcalf AK, Potter JF, Myint PK. Effect of dysphasia and dysphagia on inpatient mortality and hospital length of stay: a database study. J Am Geriatr Soc 2009; 57:2101-2106.

2. Martin BJ, Corlew MM, Wood H, et al. The association of swallowing dysfunction and aspiration pneumonia. Dysphagia 1994;9:1-6.

3. Lee TH, Lee JS, Kim WJ. High resolution impedance manometric findings in dysphagia of Huntington's disease. World J Gastroenterol 2012;18:1695-1699.

4. Lee TH, Lee JS. High-resolution Manometry for Oropharyngeal Dysphagia in a Patient With Large Cervical Osteophytes. J Neurogastroenterol Motil 2012;18:338-339.

5. Omari TI, Dejaeger E, van Beckevoort D, et al. A method to objectively assess swallow function in adults with suspected aspiration. Gastroenterology 2011;140:1454-1463.

6. Omari TI, Ferris L, Dejaeger E, Tack J, Vanbeckevoort D, Rommel $\mathrm{N}$. Upper esophageal sphincter impedance as a marker of sphincter opening diameter. Am J Physiol Gastrointest Liver Physiol 2012; 302:G909-913.

7. Noh EJ, Park MI, Park SJ, Moon W, Jung HJ. A case of amyotrophic lateral sclerosis presented as oropharyngeal Dysphagia. J Neurogastroenterol Motil 2010;16:319-322.

8. Mielens JD, Hoffman MR, Ciucci MR, Jiang JJ, McCulloch TM. Automated analysis of pharyngeal pressure data obtained with high- resolution manometry. Dysphagia 2011;26:3-12.

9. Umeki H, Takasaki K, Enatsu K, Tanaka F, Kumagami H, Takahashi $\mathrm{H}$. Effects of a tongue-holding maneuver during swallowing evaluated by high-resolution manometry. Otolaryngol Head Neck Surg 2009;141:119-122.

10. Takasaki K, Umeki H, Enatsu K, et al. Investigation of pharyngeal swallowing function using high-resolution manometry. Laryngoscope 2008;118:1729-1732.

11. Pal A, Williams RB, Cook IJ, Brasseur JG. Intrabolus pressure gradient identifies pathological constriction in the upper esophageal sphincter during flow. Am J Physiol Gastrointest Liver Physiol 2003; 285:G1037-1048.

12. Eisenhuber E, Schima W, Schober E, et al. Videofluoroscopic assessment of patients with dysphagia: pharyngeal retention is a predictive factor for aspiration. AJR Am J Roentgenol 2002;178:393-398.

13. Omari TI, Dejaeger E, Van Beckevoort D, et al. A novel method for the nonradiological assessment of ineffective swallowing. Am J Gastroenterol 2011;106:1796-1802.

14. Omari TI, Dejaeger E, Tack J, Vanbeckevoort D, Rommel N. An impedance-manometry based method for non-radiological detection of pharyngeal postswallow residue. Neurogastroenterol Motil 2012; 24:e277-e284.

15. Lee TH, Lee JS, Park JW, et al. High-resolution impedance manometry facilitates assessment of pharyngeal residue and oropharyngeal dysphagic mechanisms. Dis Esophagus 2014;27:220-229.

16. Warnecke T, Teismann I, Oelenberg $\mathrm{S}$, et al. Towards a basic endoscopic evaluation of swallowing in acute stroke - identification of salient findings by the inexperienced examiner. BMC Med Educ 2009; 9:13.

17. Szczesniak MM, Rommel N, Dinning PG, Fuentealba SE, Cook IJ, Omari TI. Optimal criteria for detecting bolus passage across the pharyngo-oesophageal segment during the normal swallow using intraluminal impedance recording. Neurogastroenterol Motil 2008;20: 440-447.

18. Blonski W, Hila A, Vela MF, Castell DO. An analysis of distal esophageal impedance in individuals with and without esophageal motility abnormalities. J Clin Gastroenterol 2008;42:776-781.

19. Kelly AM, Drinnan MJ, Leslie P. Assessing penetration and aspiration: how do videofluoroscopy and fiberoptic endoscopic evaluation of swallowing compare? Laryngoscope 2007;117:1723-1727.

20. Kelly AM, Leslie P, Beale T, Payten C, Drinnan MJ. Fibreoptic endoscopic evaluation of swallowing and videofluoroscopy: does examination type influence perception of pharyngeal residue severity? Clin Otolaryngol 2006;31:425-432.

21. Landis JR, Koch GG. The measurement of observer agreement for categorical data. Biometrics 1977;33:159-174.

22. Hoffman MR, Ciucci MR, Mielens JD, Jiang JJ, McCulloch TM. Pharyngeal swallow adaptations to bolus volume measured with high-resolution manometry. Laryngoscope 2010;120:2367-2373. 